\section{Depending on GHSR}

\section{By Michael J. Haas, Senior Writer}

Although a handful of biotech companies are pursuing ghrelin antagonists to treat obesity, the hormone target could also have a role in alcohol dependence, according to a team led by Swedish researchers. ${ }^{1}$ Even though the findings reveal a new indication for ghrelin inhibitors, it's still unclear whether the molecules could treat alcoholism without altering appetite or body weight.

Ghrelin plays a role in regulating appetite and energy homeostasis in the body. Compounds that block its receptor, growth hormone secretagogue receptor (GHSR), are in development to treat obesity, whereas ghrelin agonists are being pursued to treat cachexia and postoperative ileus (POI).

Ghrelin is also thought to play a role in the brain's reward system, which motivates behaviors by inducing pleasurable effects. Thus, Suzanne Dickson and Jörgen Engel of the University of Gothenburg wanted to know whether ghrelin signaling was involved in the reward that drives alcohol dependence.

Dickson is a professor of physiology and neuroendocrinology, and Engel is professor emeritus of pharmacology.

They injected ghrelin into a region of the midbrain of mice and saw greater alcohol consumption in normal animals but not in Ghsr knockout mice or those treated with GHSR inhibitors.

Ghsr knockout or GHSR inhibition also reduced behaviors associated with reward of injected alcohol in three different mouse models of alcohol dependence. Because the mice were made alcohol dependent by alcohol injection - not consumption - the results showed that the role of ghrelin signaling in alcohol reward was unrelated to alcohol's caloric value and thus distinct from ghrelin's role in regulating appetite, the team wrote in their report in the Proceedings of the National Academy of Sciences.

The team also wrote that the two GHSR-targeting compoundsJMV2959 (AEZS-123) from Aeterna Zentaris Inc. and BIM28163 from Ipsen Group - had opposite effects on food intake of the mice: AEZS-123 decreased it and BIM28163 increased it. This provided further evidence that the role of ghrelin signaling in alcohol reward was independent of alcohol's caloric value, according to Elisabet Jerlhag, lead author of the PNAS study and a postdoctoral fellow at The Sahlgrenska Academy at the University of Gothenburg.

Ipsen researchers had previously reported that BIM28163 increased food intake, a result that the company attributed to the possibility that a receptor other than GHSR mediated ghrelin's actions on feeding and weight gain. ${ }^{2}$

According to Rakesh Datta, global discovery project leader at Ipsen and coauthor on the PNAS study, the company had discovered BIM28163 as part of its preclinical obesity program but discontinued development because of the compound's effects on appetite.

The company's lead compound, the GHSR agonist BIM28131, is in IND-enabling preclinical studies to treat gastrointestinal transit disorders, said CSO Jacques-Pierre Moreau. The company has not announced a start date for Phase I trials.

He added that the company also plans to develop the compound to treat cachexia.

Aeterna Zentaris has GHSR ligands in preclinical development to treat obesity and related endocrine disorders but would not disclose whether AEZS-123 is among those compounds. The company also declined to disclose whether it would develop AEZS-123 to treat alcohol dependence and also declined to characterize the compound.

Besides researchers from Gothenburg, Aeterna Zentaris and Ipsen, the PNAS team included researchers from Johnson \& Johnson and the National Institute on Alcohol Abuse and Alcoholism.

\section{Food and drink think}

Although the PNAS study provides a rationale for developing GHSR inhibitors to treat alcohol dependence, companies wanted to see experiments on the effects of GHSR inhibition on appetite and on other models of alcoholism.

“The PNAS findings provide preliminary support for the idea that inhibiting ghrelin receptors in the central nervous system could block the behavioral and neurochemical effects of alcohol intake," said Conrad Wong, clinical research advisor in medical neuroscience at Eli Lilly and Co.

William Mann, COO of Helsinn Healthcare S.A.'s Helsinn Therapeutics U.S. Inc. unit, also thinks the PNAS paper "provides early evidence for an antighrelin approach to treating alcohol dependence."

Mann wants to see additional work to determine whether GHSR can be targeted without adversely affecting appetite or food intake.

Helsinn has two small molecule ghrelin mimetics in development. Anamorelin (RC-1291) is in Phase II testing treat cachexia in chemotherapy patients. Ipamorelin is in Phase II testing to treat POI. The company obtained both compounds with its acquisition of Sapphire Therapeutics Inc. in January.

Jerlhag acknowledged that the PNAS team's results showed that changes in food intake were potential side effects of targeting GHSR to treat alcohol dependence. But she noted that mice treated with AEZS-123 did not exhibit a pronounced reduction in food intake or any difference in body weight compared with untreated controls over the course of five days. 


\section{TARGETS \& MECHANISMS}

Wong said further studies need to evaluate the effect of GHSR inhibition on other measures of motivations for alcohol consumption besides locomotor stimulation and dopamine release in the forebrain, which were tested by the PNAS team. "The alcohol use disorder population is highly heterogeneous and will require several novel medications that address various phenotypes of this disease state" such as binge drinking, chronic heavy drinking and psychiatric comorbidities, he said.

Lilly has two compounds in Phase II testing to treat alcohol dependence: LY676017, a neurokinin 1 (NK1) antagonist, and LY2196044 (OpRA II), which has an undisclosed target.

In addition to the challenges of a heterogeneous population, the study of alcohol use disorders is complicated by the likelihood that multiple mechanisms are at work. A case in point is a report by another multinational team in The Journal of Neuroscience earlier this month, which described a signaling pathway in the brain-involving metabotropic glutamate receptor subtype 5 (mGluR5), homer homo$\log 2$ (HOMER2; CPD) and phosphoinositide 3-kinase (PI3K) - that regulated binge drinking in mice. ${ }^{3}$

Karen Szumlinski, leader of the study and assistant professor of psychology at the University of California, Santa Barbara, said there was no known link or overlap between the two signaling pathways described in the PNAS and Journal of Neuroscience articles. Jerlhag and Dickson agreed.

Szumlinski's team included researchers from The Johns Hopkins University School of Medicine, Oregon Health \& Science University, the Portland VA Medical Center, the Polish Academy of Sciences and the Johannes Gutenberg University Mainz.

Jerlhag said the PNAS team is investigating the role of ghrelin signaling in reward associated with other drugs of abuse such as nicotine, cocaine and amphetamines. The findings reported in PNAS are patented, she told SciBX.

Haas, M.J. SciBX 2(29); doi:10.1038/scibx.2009.1131

Published online July 30, 2009

\section{REFERENCES}

1. Jerlhag, E. et al. Proc. Natl. Acad. Sci. USA; published online June 29, 2009; doi:10.1073/pnas.0812809106

Contact: Elisabet Jerlhag, University of Gothenburg, Gothenburg, Sweden e-mail: elisabet.jerlhag@pharm.gu.se

2. Halem, H. et al. Eur. J. Endocrinol. 151, S71-S75 (2004)

3. Cozzoli, D. et al. J. Neurosci. 29, 8655-8668 (2009)

\section{COMPANIES AND INSTITUTIONS MENTIONED}

Aeterna Zentaris Inc. (TSE:AEZ; NASDAQ:AEZS), Quebec City, Quebec, Canada

Eli Lilly and Co. (NYSE:LLY), Indianapolis, Ind.

Helsinn Healthcare S.A., Pazzallo, Switzerland Ipsen Group (Euronext:IPN), Paris, France

Johannes Gutenberg University Mainz, Mainz, Germany

The Johns Hopkins University School of Medicine, Baltimore, Md. Johnson \& Johnson (NYSE:JNJ), New Brunswick, N.J.

National Institute on Alcohol Abuse and Alcoholism, Bethesda, Md. Oregon Health \& Science University, Portland, Ore.

Polish Academy of Sciences, Krakow, Poland Portland VA Medical Center, Portland, Ore. University of California, Santa Barbara, Calif. University of Gothenburg, Gothenburg, Sweden 